\title{
Parental opinion of consent in Neonatal Research
}

Karen McCarthy1, Niamh Ryan ${ }^{1}$ Darragh O'Shea ${ }^{1}$, Kieran Doran 1, Richard Greene 2, Vicki Livingstone², Anthony Ryan¹, Geraldine Boylan ${ }^{1,2}$, Eugene Dempsey ${ }^{1,2}$

${ }^{1}$ Department of Paediatrics and Child Health, Cork University Maternity Hospital, Wilton, Cork, Ireland 2INFANT, Irish Centre for Fetal and Neonatal Translational research, University College Cork, Ireland

\section{Introduction}

- Obtaining valid informed consent can be challenging in neonatal research.

- Provision of information and allowing parents time to process and understand can be difficult.

- Alternative proposed consent processes include antenatal consent, deferred, waived, or a continuous consent process,. all of which have their own potential limitations.

- Aim: To determine parental perceptions of the consent process in neonatal research and to explore acceptability of methods used to obtain consent.

\section{Method}

- A parental questionnaire was developed to examine parental attitudes to consent and hypothetical research scenarios.

- Population: Parents attending outpatient clinics and postnatal inpatients were invited to participate.

- Four hypothetical studies were presented and parents were asked about different methods of obtaining consent.

\section{Results}

- 600 responses.

- Mean (SD) parental age was 32.4 (5.3) years.

- Majority had completed third level education (75\%)

- $72 \%$ were mothers.

- 112 (19\%) had previously been approached to participate in a research study and 101 (90\%) consented to on that occasion.

- $40 \%(n=238)$ felt neonates involved in research received better overall care.

- In emergency research 311 (52\%) felt that full informed consent was necessary while 132 (22\%) were unsure.

- $75 \%$ of parents would prefer to be approached to discuss potential research studies before birth.

- Parental responses to hypothetical research scenarios are presented in table 1.

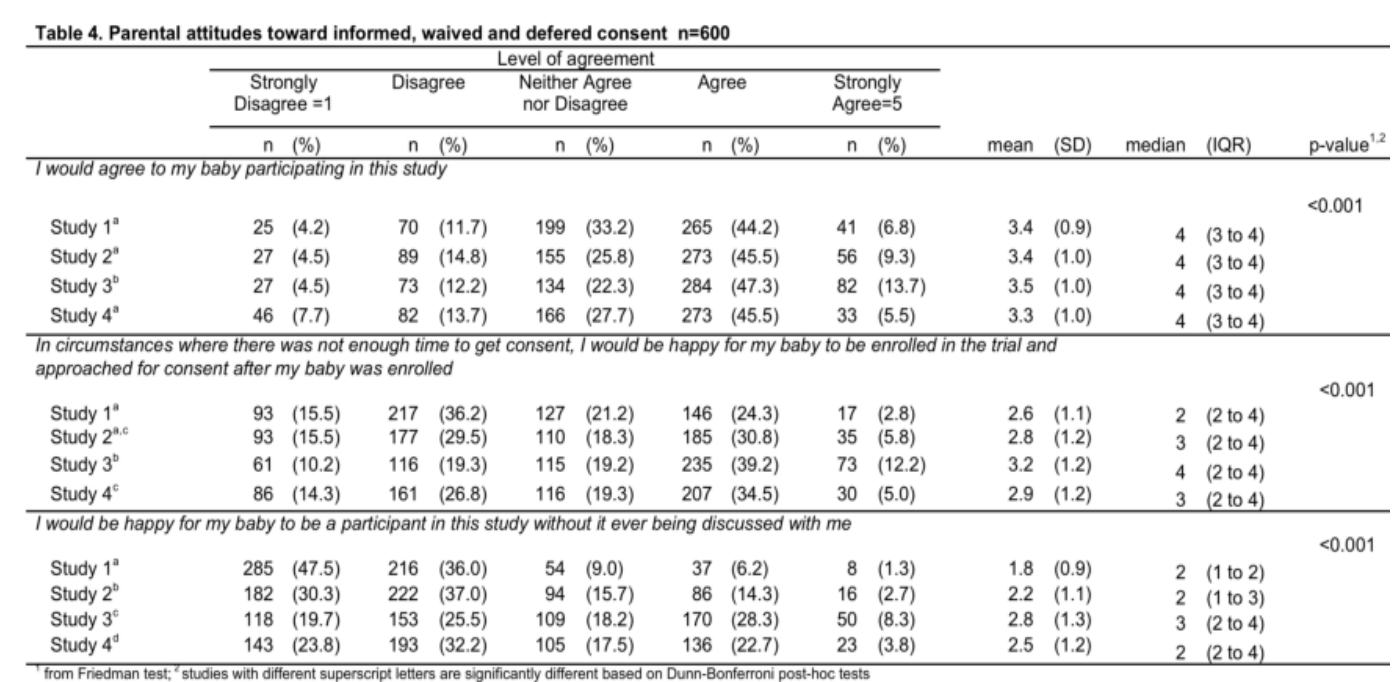

\section{Discussion}

- The majority of parents would like to be approached to discuss research studies in the antenatal period.

- Overall parents were most likely to consent to study 3 ('resuscitation study') and least likely to consent to study 1 ('feeding study').

- Parents may be more likely to consent to higher risk research where infants are more acutely unwell but where the potential benefits of improving treatment are much greater.

- Strongest agreement was for informed consent, followed by deferred with the lowest agreement for waived consent in all four studies.

- Deferred and waived consent were less acceptable in non-urgent situations.

- Parents were more willing to accede to deferred or waived consent in the time sensitive study.

\section{Conclusion}

Parents believe informed consent is an important pre-requisite to neonatal research. Current consent methods are undermined by difficulties with timing and the vulnerability of parents in the perinatal period. Parents in this study demonstrate understanding of the challenges surrounding informed consent in emergency research. 